Urol. internation. 1956;3:395-396

\title{
Register rerum ad Vol. 3
}

Bearbeitet von W. Baumann, Basel

Adnextuberkulose, Bchandlung mit Tu-berkulin, 107

Antibiotic treatment of renal tuberculosis, 39

Atonie myogénique de la vessie, 304

Bassinet, la r円esure de la pression, 233 Bladder, myogenous atony, 304 Blase, myogene Atonie, 304 Blasenhalsadenom siehe Prostatahyper-

trophie Blood level of PAS and Streptomycine,

114 Blutspíegel von PAS und Streptomycin,

114

Ceinture artérielle péri-pyélique, 14 Chemotherapie der Genitaltuberkulose, 162, 170 Chemotherapie der Nierentuberkulose,

Hístologie, 144 Chemotherapie der Urogenitaltuberku-

lose, 39, 66, 80, 90, 96, 110, 114 Cholecystopathy, tonus in the urinary

tract, 1 Cysto-urétéroplastie, 175, 223 Colique néphrétique, tonus des voies

urinaires, 1 Cushing-Syndrom bei Chemotherapie,

106

Druckmessung mit Kathetern, 233

Electrolyte imbalance in prostatic hypertrophy, 311 Ersatzblase, 25

Fibrinolysin der Prostata, 273 Fistules urétérales, traitement, 175, 223

Genitaltuberkulose, Chemotherapie, 162, 170

Harnstauung und Nierenschaden, 357 Histamine, son efFet sur la circulation

rénale, 56 Histopathology of renal tuberculosis

after chemotherapy, 144 Hydronephrosis, extrarenal, 14

Hydronephrosis, conservative surgical

treatment, 190 Hypertrophie prostatique v. prostatic

hypertrophy Incontinence orthostatique chez la

femme, 329 Incontinence relative chez la femme,

traitement opératoire, 325 Kidney, circulatory effect of Histamine,

56 Nekrosperrnie bei chron. Prostatitis, 273 Nephralgia, 1

Néphrectorníe partieile, 126, 144 Nierenbeckendruck, Messung mit Kathetern, 233

Nierendurchblutung, Histamineffekt, 56 Nierenkolik, Tonus im Harntrakt, 1 Nierentuberkulose siehe Urogenital-

tuberkulose Pathologic anatomy of renal tuberculosis after chemotherapy, 144 Phosphatase,

saure, 273 Polresektion der Niere bei Tuberkulose,

126, 144 Polype de Гurtère pelvien, traitement,

175 Pression au niveau du bassinet, 233 Pressure in the renal pelvis, 233 Prostata, corpora

amylacea, 273 Prostata, Physiologie, 261 Prostata, vergleichende Morphologic,

251 Prostata und Prostatahypertrophie, $\mathrm{Pa}-$

thophysiologie, 273 Prostataatrophie, 273 Prostatahypertrophie, klinische Fragen, 
304 Prostatahypertrophie, konservative Behandlung, 297, 304 Prostatahypertrophie, Tonus des Harntraktes, 1 Prostatahypertrophie, Pathophysiologie, 273 Prostata-Massage, 273

Prostatatuberkulose, endoskopische Re-sektion, 170

Prostate, la morphologie comparée, 251

Prostate, la physiologie, 261

Prostate et hypertrophie prostatique, physiologie pathologique, 273

Prostatic adenoma v. prostatic hypertrophy

Prostatic hypertrophy, clinical questions, 304

Prostatic hypertrophy, conservative treatment, 297

Prostatic hypertrophy, electrolytes, 311

Prostatic hypertrophy, tonus in the urinary tract, 1

Prostatic tuberculosis, transurethral resection, 170

Pyélectasie, 14

Pyelitis und Nierenschaden, 357

Pyelogramm, Tonuszunahme des Harn-traktes, 1

Pyelonephritic contracted kidney, pa-thogenesis, 357

Pyelonephritic infection and nephro-sclerosis, 357

Rein rétracté, etudes expérimentales sur la pathogénie, 357

Renal colic, tonus in the urinary tract, 1

Renal pelvis, measuring of pressure, 233

Renal tuberculosis, long-term treatment, 39, 66, 80, 90, 96, 110, 114

Samenuntersuchung bei Urogenital-tuberkulose, 162

Schrumpfblase, operative Behandlung, 25

Schrumpfhiere, Pathogenese der pyeli-tischen -, 357

Semen, guinea-pig tests in genital tuberculosis, 162

Sperme, inoculation en cas de tubercu-lose génitale, 162

Spermin, Spermidin, 273

Spermien und Prostatasekret, 273

Sténose urétérale, traitement, 175

Sterilität bei chron. Prostatitis, 273

Stress incontinence in the female, operative suggestion, 325

Stricture uréthrale, traitement, 243 Strictures of the pelvic ureter, treatment, 175

Taux sanguin de PAS et de streptomy-cine, 114

Tonuszunahme des Harntraktes, 1

Tonus increase in the urinary tract, 1

Tuberculose génitale, chimiothérapie, 162, 170

Tuberculose prostatique, resection endoscopique, 170

Tuberculose urogénitale, chimiothérapie, 39, 66, 80, 90, 96,110, 114

Tuberculose urogénitale, nephrectomie partielle, 126, 144

Upper urinary tract, tonus, 1, 233 Ureter, bladder flab as a substitute -,

175 Ureter, tumor of pelvic -, treatment, 175 Ureteral fistulas, treatment, 175, 223

Ureterimplantationen in Darm und

Blase, 25 Uretero-ileo-cystostomy, 223 Ureterolyse, 25

Urétéro-plastie par lambeau vésical, 175 Ureterplastik nach operativer Verlet- 
zung, 25 Ureterverletzungen, Behandlung operativer-, 175, 223 Ureterstenose, operative Behandlung,

175 Urethral stricture, treatment, 243 Urethroskop nach Fischer, 243 Urinary incontinence in the female, 325 ,

329 Urogenitaltuberkulose, Chemotherapie,

$39,66,80,90,96,110,114$ Urogenitaltuberkulose, partielle Ne-

phrektomie, 126, 144 Urography, intravenous, 1

Vessie, atonie myogénique, 304

Voies urinaires supérieures, tonus, 1 ,

233 Wertheim'sche Operation, urologische

Komplikationen, 25

COLLABORATORES

V. AALKJAER, Aarhus

S. ADANJA, Beograd

R. ALCALA-SANTARELLA,

Valencia G. E. ALKEN, Homburg/Saar M. ALVAREZ, Habana N. ALWALL, Lund R.

ANDERSEN, Oslo J. C. ANDERSON, Sheffield O. ARIAS, Mexico R. AUVIGNE, Nantes W.

BAKER, Chicago, Ill.

D. BAND, Edinburgh

F. BAQUERO-GONZALEZ,

Caracas J. BEDRNA $\uparrow$, Hradec Kralové

E. BLEGEN, Oslo

G. BIEDERMANN, InnsbruckP. BISCHOFF, Hamburg

J. BITSCHAI, Alexandrien

R. BLANGEY, Zurich

A. BORJAS, Caracas

K. BOSHAMER, Wuppertal

J, P. BOURQUE, Montreal

L. M. BREA, Buenos Aires

P. BRUNI, Napoli

E. BURNS, New Orleans, La.

G. CARROL, St. Louis, Mo.

E. CASTRO, Mexico

E. P. CASTRO, Madrid

J. M. CERVONI, Caracas

C. CHIAUDANO, Torino

A. CHRISTENSSEN,

København J. CIBERT, Lyon L. CIFUNTES DELATTE,

Madrid V. LE CLERC DANDOY,

Bruxelles O. CORREA FUENZALIDA,

Santiago E. da COSTA MANSO,

São Paulo C. D. CREEVY, Minneapolis,

Minn. J. A. CURRIE, Capetown R. DARGET, Bordeaux R. DEFOORT, Anvers J. P. DEL

POBIL, Seviila T. DE LA MAZA SAAVEDRA,

Madrid C. L. DEMING, New Haven, 
Conn. P. DENNEHY, Johannesburg M. DEROT, Paris P. DONKER, Rotterdam H. DONOVAN, Birmingham J. L. EMMETT, Rochester,

Minn. R. L. ENGELKING, Mexico

B. S. ERDURAN, Istanbul

A. G. VAN ESSEN, GroningenP. FABRE, Toulouse

B. FEY, Paris

R. H. FLOCKS, Iowa City, Iowa H. FONTOURA MADUREIRA,

Lisboa J. FORET, Liège F. FRANZAS, Kotka W. W. GALBRAITH, Glasgow A. E. GARCIA, Buenos Aires

F. GARCIA RUIZ, MexicoP. GAUSA, Barcelona

Th. E. GIBSON, San Francisco, Calif.

G. GIERTZ, Stockholm

F. de GIRONCOLI, Firenze

H. U. GLOOR, Zurich

VV. GRÉGOIR, Bruxelles

J. HAMBURGER, Paris

J. H. HARRISON, Boston, Mass.

O. HENNIG, München

H. HENNINGER, Wien

A. B. HEPLER, Seattle, Wash.

R. HERBST, Graz

K. HEUSCH, Aachen

A. HIDALGO, Madrid

C. C. HIGGINS, Cleveland, OhioK. HOEG, Oslo

R. S. HOTCHKISS, New York H. HOUTAPPEL, Amsterdam F. HUEDEPOHL, Berlin T.

INADA, Kyoto A. INGELMAN-SUNDBERG,

Stockholm Z. KAIRIS, Athen J. KELLER, Dresden VV. K. KERR, Toronto K. KIRKLAND, Sydney M. KLIKA, Praha T. G. KNUDTZON, København O. KRIVEC, Zagreb T.

KUSUNOKI, Niigata R. KUSS, Paris T. J. D. LANE, Dublin J. P. LEGAULT, Montreal C.

LOBO ONELL, Santiago J. LOMELIN, Mexico V. F. MARSHALL, New York R. I. MATHIS,

Buenos Aires VV. MATHISEN, Oslo S. McMAHON, Durban A. Q. MENDIZABAL, Mexico F. von MIKULICZ-RADECKI,

Berlin J. MOMBAERTS, Bruxelles VV. A. MOONEN, Vught T. D. MOORE, Memphis, Tenn.

R. M. NESBIT, Ann Arbor, Mich.

G. NICOLICH, Genova

R. NISSEN, Basel

V. J. O'CONOR, Chicago, Ill.

S. OHMORI, Chiyodaku, Tokyo

0. OLSSON, Lund

1. ORSOLA-MARTI, BarcelonaV. PACES, Praha

M. PAVONE, Palermo

R. PEDROSO, Habana

CH. PERRIER, Geneve

M. E. PESQUEIRA, Mexico

J. PICATOSTE Y PICATOSTE,

Santander L. PISANI, Milano 
D. POOLE-VVILSON, ManchesterG. C. PRATHER, Boston, Mass.A. PUIGVERT, Barcelona

L. N. PYRAH, Leeds

S. RAKOVEC, Ljubljana

G. RAVASINI, Padova

L. REBAUDI, Buenos Aires

J. M. RETIEF, Capetown

F. REUBI, Bern

C. J. ROBSON, Toronto, Ontario

R. ROCHA BRITO, São Paulo

L. H. RODRIGUEZ-DIAZ,

Caracas L. RODRIGUEZ-SANTANA,

Caracas C. RUSCHE, Los Angeles, Calif. A. SADI, São Paulo R. E. SANDRO, Buenos Aires L. SANJURJO, Santurce/Puerto

Rico H. J. SARRE, Freiburg i.Br. M. SECRÉTAN, Lausanne F. SERRALLACH JULIA,

Barcelona H. M. SPENCE, Dallas, Texas R. STEINERT, Oslo C. B. STEWART, Winnipeg,

Manitoba M. STOLZE, Halle C. J. SVENDSEN, Oslo T. St. TANEFF, Sofia

E. THIERMANN, ErlangenJ. THOMA, LuxembourgL. F. TORRES, Manila

A. TRABUCCO, Buenos Aires

K. TRAUTNER, København

E. TRUC, Montpellier

P. I. TUÖVINEN, Helsinki

K. TZSCHIRNTSCH †, Iserlohn

R. ÜBELHÖR, Wien

J. H. J. VAN DER VUURST

DE VRIES, Utrecht S. GIL VERNET, Barcelona E. WILDBOLZ, Bern M. ZAMITH, Coimbra

R. ZENKER, Marburg

TRANSLATORES

English-French:

W. GRÉGOIR, 58, Rue Saint-Bernard, Brussels

(Belgium)

English-German:

H. G. STOLL, Chir. und Urolog. Klinik der Stãdt.

Krankenanstalten, Wuppertal-Barmen (Germany)

Deutsch-Englisch:

F. R. MURAD, Brewster,

Mass. (USA)

Français-A Пemand:

P. BISCHOFF, Heilwigstr. 28, Hamburg 20

(Allemagne)

Français- $\Lambda$ nglais:

J.-P. LEGAULT, 300 Carre Saint-Louis

Montreal (Canada)

Deutsch-Französisch:

G. MAYOR

Goethestr. 22 
Zurich (Schweíz) 